\title{
FIB-TEM CHARACTERIZATION OF SURFACE AND SUB-SURFACE DEFECTS INTRODUCED INTO LITHIUM NIOBATE BY A FEMTOSECOND LASER
}

\author{
E.A. Stach ${ }^{1}$, V.R. Radmilovic ${ }^{1}$, D. Deshpande ${ }^{2}$, A. Malshe ${ }^{2}$, D. Alexander ${ }^{3}$, D. Doerr ${ }^{3}$ \\ 1 National Center for Electron Microscopy, Lawrence Berkeley National Laboratory, Berkeley, CA 94720 \\ 2 SERC for Durable Micro and Nano Systems, Department of Mechanical Engineering, University of Arkansas, \\ Fayetteville, AR \\ 3 Center for Electro - Optics, University of Nebraska, Lincoln, NE
}

One proposed methodology for permanent optical storage is to write 'data bits' directly into transparent media through the use of femtosecond laser processing, with each induced damage spot being equivalent to one bit. ${ }^{1-4}$ Because extremely high storage densities are being targeted, it is essential to characterize the microstructure of the induced damage spots after laser processing.

In this work, we have utilized the Focused Ion Beam (FIB) in-situ lift-off technique ${ }^{5}$ to prepared samples from both surface and sub-surface damage spots and conventional and analytical transmission electron microscopy to characterize the microstructure. A femtosecond laser setup was used to process surface and subsurface structures in lithium niobate in the form of three dimensional arrays of damage spots, spaced $5 \square \mathrm{m}$ apart both laterally and in depth. ${ }^{6}$

As shown in Figure 1, the use of the FIB lift-out technique is highly appropriate to study defect geometries of this type, as it allows creation electron transparent samples of specific regions of the sample, both at the surface as well as below the surface. In Figure 2, a high magnification bright field image of the surface defect shown in the middle of Figure 1 is presented. The laser processing has ablated an approximately $2 \mu \mathrm{m}$ diameter trench in the top surface of the sample. This trench is surrounded by an amorphous region (confirmed by electron diffraction, not shown) that is approximately $100 \mathrm{~nm}$ in thickness, surrounded by an additional region of heavily defective material. Energy dispersive x-ray spectroscopy (EDS) shows that the amorphous region is deficient in oxygen.

In Figure 3, a sub-surface damage region is shown. Here, the laser processing has again ablated a significant region of the sample, but in this case the result is a void within the sample. This void is again surrounded by an amorphous region that is significantly thicker in size. EDS across this amorphous region shows that this region is has a higher relative concentration in oxygen, in contrast to the observations for the surface features. This may be a result of the high pressure inside the void when it is created - this high pressure may result in significant densification of the material at the void perimeter.

Within the presentation, we will discuss how these FIB-TEM observations correlate with ongoing molecular dynamics simulations of femtosecond laser interactions with materials. ${ }^{7,8}$

\section{References:}

[1] Glezner, Milosavljevic et al, Optics Letters 21 (1996) pp2023-2025.

[2] Qiu, Miura et al, Japanese Journal of Applied Physics 37 (1998) pp 2263-2266.

[3] Takita, Yamamoto et al, CLEO-Technical Digest B (2000), pp I232-I233.

[4] Borowiec, Mackenzie et al, Applied Physics A 76A (2003), pp 201-207.

[5] Prenitzer, Giannuzzi et al, Metal. and Mater. Trans. 29A, September 1998, pp 2399-2406.

[6] Laser processing conditions used: Wavelength: $800 \mathrm{~nm}$, Pulsewidth: 300 femtoseconds, Repetition Rate: $67 \mathrm{~Hz}$, N.A. of microscope objective: 0.4, Pulse - to - pulse stability $\sim 4 \%$ peak to peak, Energy density: $123.52 \mathrm{~J} / \mathrm{cm}^{2}$.

[7] Deshpande, et al., submitted, 2003.

[8] This work was supported by the U.S. Department of Energy under Contract No. DE-AC03-76SF00098. 

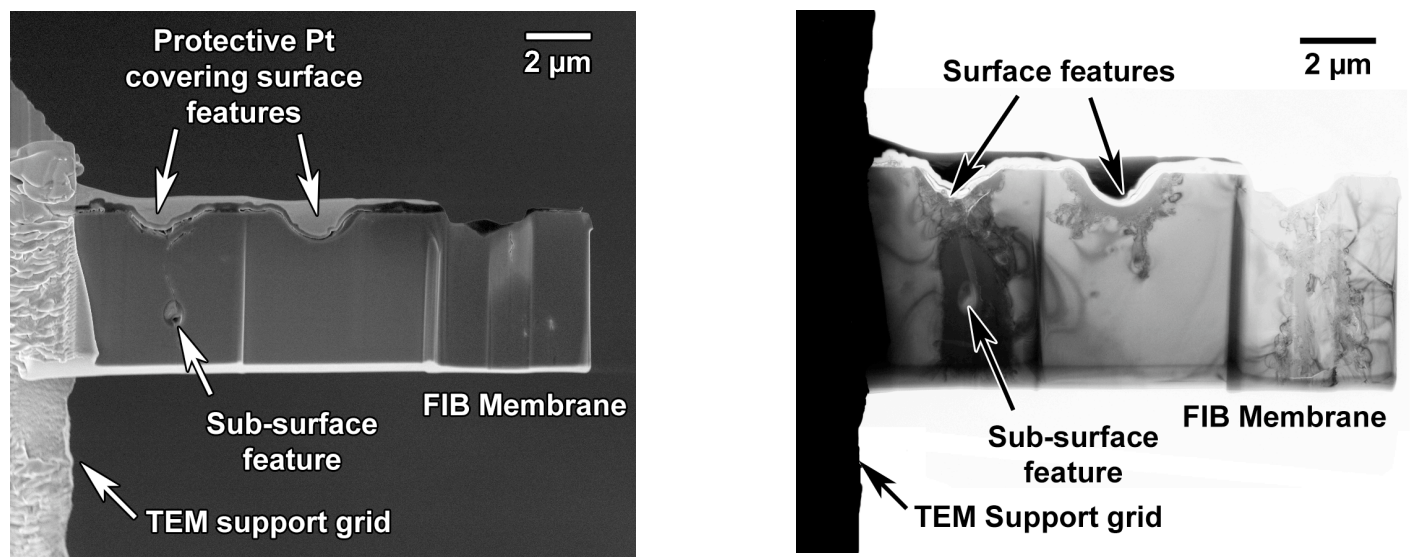

Figure 1 - (a) Secondary electron image of FIB prepared TEM specimen and (b) Bright field transmission electron micrograph of the same. In each, the presence of both the surface damage spots and sub-surface features can be seen.
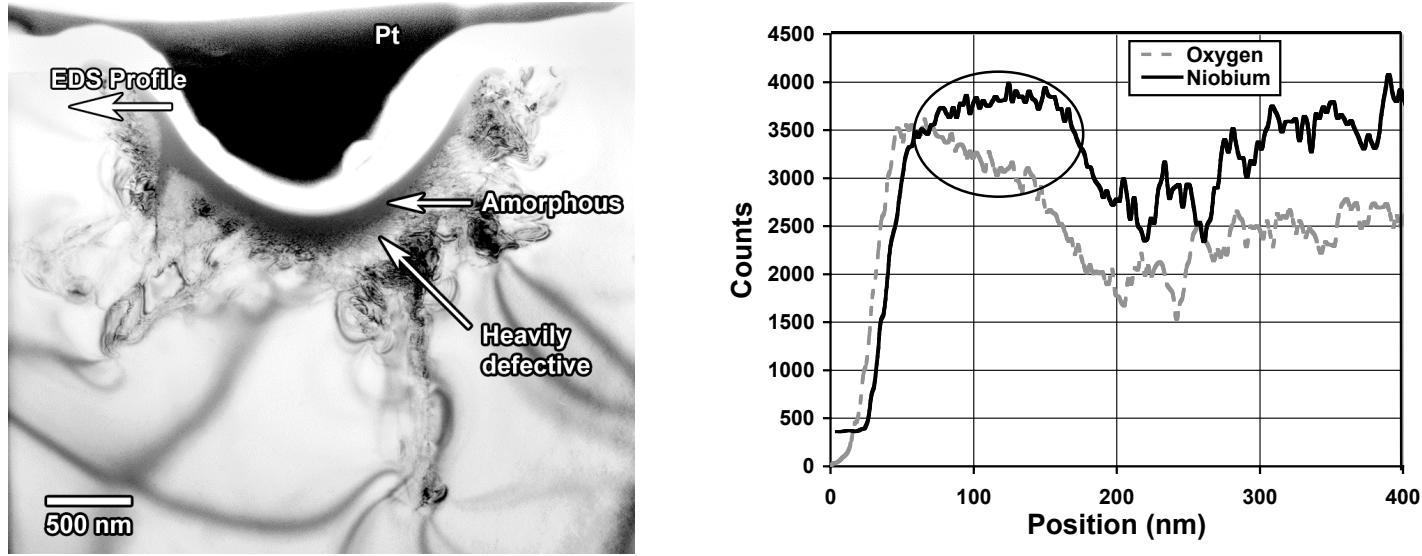

Figure 2 - (a) Bright field TEM image showing the presence of an amorphous layer of approx 100 $\mathrm{nm}$ in thickness surrounded by a heavily defective region. (b) EDS line profile showing evidence of sub-stoichiometric oxygen content in the amorphous region.
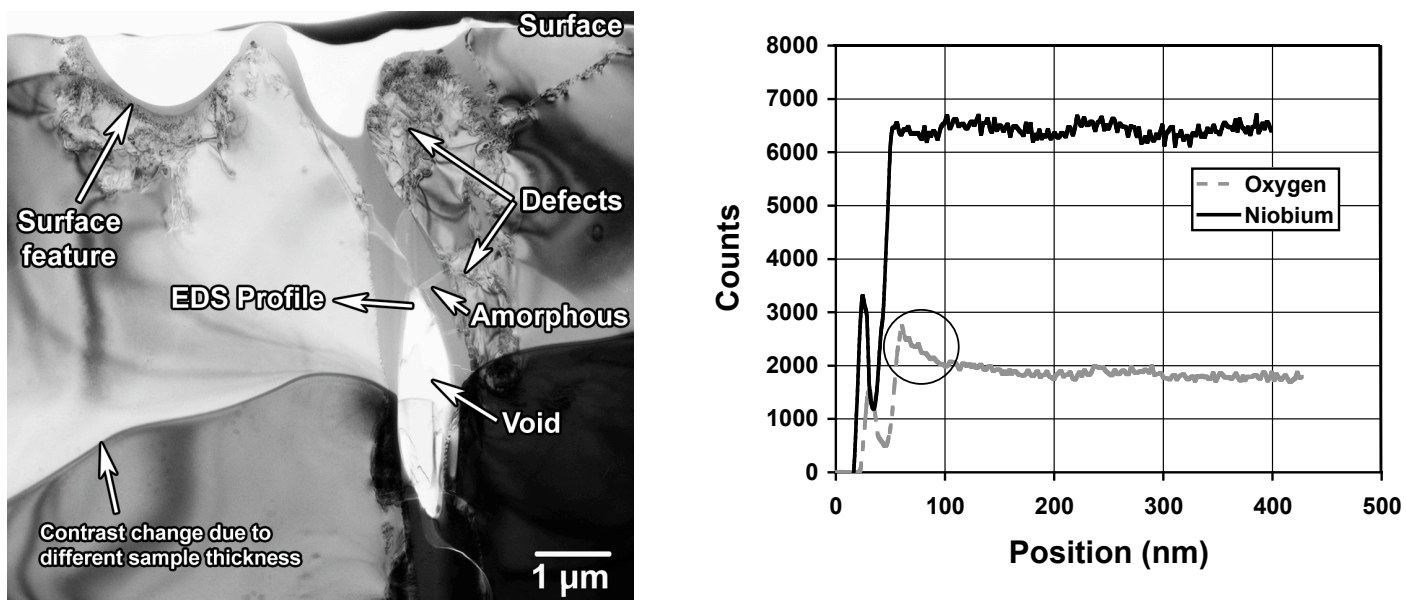

Figure 3 - (a) Bright field TEM image of both a surface and sub-surface defect (from a different sample than that shown in Figure 1). (b) EDS line profile showing excess content in the amorphous region. 